\title{
Recurrent Kaposi Sarcoma of the Ear in an HIV-negative patient: A Case report with review of the literature. Is ear a predilection site for Kaposi sarcoma in HIV-negatives?
}

\author{
ifa Etesami ${ }^{1}$, Yasamin Kalantari ${ }^{1}$, Alireza Ghanadan ${ }^{1}$, and Azadeh Rezayat ${ }^{1}$ \\ ${ }^{1}$ Tehran University of Medical Sciences
}

April 24, 2021

\begin{abstract}
Kaposi sarcoma (KS) is an angioproliferative disorder. While KS of the head and neck is common in HIV-positives, it's rare in HIV-negatives. Our case and the past 24 reported cases of ear KS reviewed here, highlight the importance of considering KS in the differential diagnosis of ear lesions in HIV-negatives.
\end{abstract}

Recurrent Kaposi Sarcoma of the Ear in an HIV-negative patient: A Case report with review of the literature. Is ear a predilection site for Kaposi sarcoma in HIV-negatives?

50 words key clinical message:

While KS of the head and neck is common in HIV-positives, it's a rare presentation in HIVnegatives. It is important to consider $\mathrm{KS}$ in the differential diagnosis of ear lesions in HIVnegative patients.

Kaposi sarcoma (KS) is an angioproliferative disorder. While KS of the head and neck is common in HIV-positives, it's rare in HIV-negatives. Our case and the past 24 reported cases of ear KS reviewed here, highlight the importance of considering KS in the differential diagnosis of ear lesions in HIV-negatives

\section{Introduction}

Kaposi sarcoma $(\mathrm{KS})$ is a rare borderline angioproliferative disorder characterized by multiple vascular mucosal or cutaneous lesions. ${ }^{1}$

It has four major types: classic (predominantly in elderly men) (CKS), African endemic (AEKS), immunosuppression associated or transplant-associated (ITKS) and AIDS-associated. ${ }^{2,3}$

The classic form typically presents with cutaneous lesions on the lower extremities. ${ }^{1}$ While the head and neck is a common site for mucocutaneous lesions in HIV patients with KS, the presence of lesions on the head and neck in HIV negative patients is a rare phenomenon. ${ }^{4,5}$

Among the reported cases of Kaposi sarcoma auricular involvement is very rare. In a study of 11 KS cases presented on head and neck, though the majority of cases were HIV-positive, the 2 patients with KS lesions on their external ears were both HIV-negative. Therefore, they highlighted the importance of considering KS as a differential diagnosis for vascular lesions on the ears of HIV-negative patients. ${ }^{6}$ This study aims to present a case of HIV-negative patient with multiple recurrent papules on his ear diagnosed as Kaposi sarcoma that developed KS lesions on his foot years later with a review of literature on KS presented on ears (table 1). 


\section{Case presentation}

A 43-year-old man first presented to our dermatology clinic in 2014 with multiple erythematous dome-shaped papules on his right auricle. He has had these lesions from 6 months before his presentation to our clinic (Fig. 1a). A biopsy was taken from his auricular papules at that time. While our most probable clinical impression was Angiolymphoid hyperplasia with eosinophilia (ALHE) or pseudolymphoma, the microscopic evaluation was consistent with KS (Fig. 2a-e). Histopathologic examination of a skin biopsy from the ear showed nodular proliferation of spindled endothelial cells arranged in intersecting fascicles with intervening slit and sieve-like vascular channels. There were some blood-filled vascular spaces between spindle cells with red blood cell extravasation and patchy infiltrate of lymphocytes and plasma cells (Fig. 2a, 2b). Some mitotic figures and apoptotic bodies were also identified. Immunohistochemistry staining reveals positive immunoreaction of tumor cells for CD31 and CD34 as well as HHV-8 which show nuclear immunoreactivity (Fig. 2c-e).

Because his lesions were limited to his ear, the lesions were totally excised (Fig. 1b). In 2018, he presented to our clinics with recurrence of one solitary papule on his right ear, the papule was totally excised and the histopathology was consistent with KS again. The patient did not come back for further evaluation at that time. In April 2020, he presented to our clinic with the recurrence of papules on his right ear and the development of an erythematous plaque on his right foot since a year ago. Two biopsies were taken from his ear and foot lesions that both were consistent with KS. Routine laboratory evaluations including complete blood count (CBC), liver and renal function tests were normal and HIV testing was negative. The patient was otherwise healthy without any history of immunodeficiency. He was not taking any medication.

\section{Discussion}

While oral (59.1\%) and craniofacial (43.9\%) involvement is common in HIV-positives(1), Kaposi sarcoma of the head and neck is rare (approximately $<5 \%$ of the KS cases) in the HIV-negative individuals. ${ }^{6}$ The most common presentation of KS in HIV-negatives is multiple bilateral lesions of the lower extremities. ${ }^{7}$ Among the head and neck KS, the incidence of auricular involvement is much lower, so it should be considered a distinct manifestation. The presence of a recurrent, auricular KS with an atypical presentation in a young immunocompetent individual is a very rare finding.

In this article, we presented a case of recurrent KS on the ear with a literature review on ear KS cases (Table 1). 5, 6, 8-27 The literature review disclosed 24 cases since the year 1941 until 2020, highlighting the rarity of this presentation. Sixteen males and seven females aged 3-85 years (median, 62 years; mean, 57.4 years) were retrieved. Of these 24 cases of ear skin KS, 2 cases were HIV-positive ${ }^{24,} 27,13$ cases were HIVnegative $\mathrm{f}^{6,16-23,25,26}$ and others were unknown. Among these, 4 cases had visceral involvement including lymph node, bone, urethra, stomach, colon, liver and spleen and the rest were limited to the skin including Just limited to the auricle $(n=11)$, ear and mucosal sites $(n=4)$, ear and extremities $(n=9)$, ear and other sites in the head and neck region (chin, eyelid) $(\mathrm{n}=3)$. Among the $13 \mathrm{HIV}$-negatives, 5 cases had some degrees of immunosuppression ( 1 case kidney transplantation ${ }^{25}, 1$ case Congenital immunodeficiency ${ }^{25}, 1$ case receiving systemic corticosteroid ${ }^{23}, 1$ case non-Hodgkin lymphoma ${ }^{21}$ and the last a case of severe lymphocytopenia ${ }^{17}$. While excision was the most common treatment option, other modalities were antiretroviral medications for HIV-positives, radiotherapy and chemotherapy with liposomal doxorubicin, bleomycin, vincristine and IFN-alpha for more widespread disease. Among 17 cases that their follow up were available, ranging from 15 months to 17 years, the majority of them were free of disease after the initial treatment $(\mathrm{n}=12), 3$ cases had recurrent lesions, 1 case was alive with disease and 1 died with disease because of uremia. While KS in HIV-negative patients has an indolent coarse, our case was highly recurrent, despite total excision with free margins, it has recurred twice in five years and after that, a new lesion on the foot appeared. So the recurrence rate of the KS in the ear needs to be further studied.

While KS pathogenesis is multifactorial and both genetic and environment are responsible, Human Herpes Virus 8 (HHV8) is the main causal factor in the development of KS in all variants irrespective of the clinicopathological setting of the disease. ${ }^{4,28}$ HHV8 contributes to cell growth, signaling apoptosis, angiogenesis, 
and immunomodulation. It produces some proteins that inhibit host adaptive and innate immunity. 1,4 While the increased risk of KS in HIV-positives and iatrogenically immunosuppressed cases is well understood, the occurrence in immunologically competent individuals remains largely unelucidated. ${ }^{7}$ Agaimy A et al hypothesized that maybe impaired local immunosurveillance and pro-inflammatory cytokines release is the causative factor. ${ }^{6}$ Although the exact reason why the ear is a predilection site in HIV negative patients who develop KS in head and neck region is not clear Francés RL et al proposed that in addition to some factors such as trauma and infection in acral sites, insufficient vascularization makes it difficult for immune system to access. ${ }^{22}$

Due to the rarity of head and neck, KS, especially in HIV-negative patients, unusual presentations of KS may be challenging if not considered in the differential diagnosis. The occurrence of KS in atypical sites like ear leads to unrecognition and misdiagnosis. The possibility of occult HIV infection should be considered beside. They may be misdiagnosed as other spindle cell tumors pathologically or other vascular lesions such as ALHE clinically. HHV8 immunohistochemistry was positive in $95 \%$ of KS lesions irrespective of HIV positivity $^{4}$, so it is a good marker to detect $\mathrm{KS}$.

In summary, we presented a case of recurrent ear KS in a young HIV-negative and otherwise healthy individual with a review of the literature on 24 cases of ear KS from 1941 to 2020 implicating ear as a predilection site for head and neck KS in HIV-negative patients, therefore, we highly suggest to consider KS as a differential diagnosis for lesions on ears.

Author 1: has made substantial contributions to conception and design, acquisition of data, analysis and interpretation of data, drafting the manuscript and revising it critically, given final approval of the version to be published. Author 2: has made substantial contributions to conception and design, acquisition of data, analysis and interpretation of data, drafting the manuscript and revising it critically. Author 3: has made substantial contributions to conception and design, drafting the manuscript and revising it critically, Author 4: has made substantial contributions to conception and design, acquisition of data, analysis and interpretation of data, drafting the manuscript and revising it critically.

\section{References:}

1. Ramirez-Amador V, Anaya-Saavedra G, Martinez-Mata G. Kaposi's sarcoma of the head and neck: a review. Oral oncology. 2010;46(3):135-45

2. Vangipuram R, Tyring SK. Epidemiology of Kaposi sarcoma: review and description of the nonepidemic variant. Int J Dermatol. 2019;58(5):538-42.

3. Cesarman E, Damania B, Krown SE, Martin J, Bower M, Whitby D. Kaposi sarcoma. Nature Reviews Disease Primers. 2019;5(1):1-21.

4. Patrikidou A, Vahtsevanos K, Charalambidou M, Valeri RM, Xirou P, Antoniades K. Non-AIDS Kaposi's sarcoma in the head and neck area. Head \& Neck: Journal for the Sciences and Specialties of the Head and Neck. 2009;31(2):260-8.

5. GNEPP DR, CHANDLER W, HYAMS V. Primary Kaposi's sarcoma of the head and neck. Annals of internal medicine. 1984;100(1):107-14.

6. Agaimy A, Mueller SK, Harrer T, Bauer S, Thompson LD. Head and neck Kaposi sarcoma: clinicopathological analysis of 11 cases. Head and neck pathology. 2018;12(4):511-6.

7. Jakob L, Metzler G, Chen K-M, Garbe C. Non-AIDS associated Kaposi's sarcoma: clinical features and treatment outcome. PLoS one. 2011;6(4).

8. Epstein E. A case for diagnosis (Kaposi's sarcoma?). Archives of Dermatology and Syphilology. 1941;34:409-10.

9. Naunton R, Stoller F. Kaposi's sarcoma of the auricle. The Laryngoscope. 1960;70(11):1535-40. 
10. Rothman S. Some clinical aspects of Kaposi's sarcoma in the European and North American populations. Acta-Unio Internationalis Contra Cancrum. 1962;18:364-71.

11. Gibbs R. Kaposi's sarcoma involving the ears. Archives of dermatology. 1968;98(1):104.

12. Howland W, Armbrecht E, Miller J. Oral manifestations of multiple idiopathic hemorrhagic sarcoma of Kaposi: report of two cases. Journal of oral surgery (American Dental Association: 1965). 1966;24(5):445-9.

13. Hardy MA, Goldfarb P, Levine S, Dattner A, Muggia FM, Levitt S, et al. De novo Kaposi's sarcoma in renal transplantation. Case report and brief review. Cancer. 1976;38(1):144-8.

14. Mikkelsen F, Nielsen N, Hansen J. Kaposi's sarcoma in polar eskimos. Acta dermato-venereologica. 1977;57(6):539-41.

15. Stearns MP, Hibbard AA, Patterson HC. Kaposi's Sarcoma of the ear: a case study. The Journal of Laryngology \& Otology. 1983;97(7):641-5.

16. Babuccu O, Kargi E, Hoşnuter M, Doğan BG. Atypical presentation of Kaposi's sarcoma in the external ear. Kulak burun bogaz ihtisas dergisi: $\mathrm{KBB}=$ Journal of ear, nose, and throat. 2003;11(1):17-20.

17. Hussein MR. Cutaneous and lymphadenopathic Kaposi's sarcoma: a case report and review of literature. Journal of cutaneous pathology. 2008;35(6):575-8.

18. Altunay I, Kucukunal A, Demirci GT, Ates B. Variable clinical presentations of Classic Kaposi Sarcoma in Turkish patients. Journal of dermatological case reports. 2012;6(1):8.

19. Colletti G, Allevi F, Moneghini L, Rabbiosi D. Bilateral auricular classic Kaposi's sarcoma. Case Reports. 2013;2013:bcr2013200059.

20. Izquierdo CM, Pérez OM, Gómez M-ZJ. Kaposi sarcoma in the external ear. Acta otorrinolaringologica espanola. 2013;64(6):448.

21. Busi M, Altieri E, Ciorba A, Aimoni C. Auricular involvement of a multifocal non-AIDS Kaposi's sarcoma: a case report. Acta Otorhinolaryngologica Italica. 2014;34(2):146.

22. Francés RL, Bouret LA, Muñoz RC, Bañuls RJ. Non-AIDS Kaposi sarcoma in the external ear. Actas dermo-sifiliograficas. 2016;107(10):872.

23. Rachadi H, Zemmez Y, Znati K, Ismaili N, Hassam B. External ear nodule revealing a disseminated Kaposi disease. Dermatology online journal. 2016;22(8).

24. Chai G, Meng Y, Liu Y, Zhao M. External auditory canal HIV-associated Kaposi sarcoma: a case report. Lin chuang er bi yan hou tou jing wai ke za zhi= Journal of clinical otorhinolaryngology, head, and neck surgery. 2018;32(24):1910-1.

25. Baykal C, Atci T, Buyukbabani N, Kutlay A. The spectrum of underlying causes of iatrogenic Kaposi's sarcoma in a large series: A retrospective study. Indian journal of dermatology. 2019;64(5):392.

26. Rupp NJ, Bode B, Broglie MA, Morand GB. Kaposi Sarcoma of the Ear in HIV-Negative Patients. Head and neck pathology. 2019;13(2):255-6.

27. McNally M, Narala S, Koshelev M. HIV patient with painless bilateral external ear nodules. JAAD Case Reports. 2020;6(3):222-4.

28. Sarid R, Klepfish A, Schattner A, editors. Virology, pathogenetic mechanisms, and associated diseases of Kaposi sarcoma-associated herpesvirus (human herpesvirus 8). Mayo Clinic Proceedings; 2002: Elsevier.

\section{Figure legends:}

Figure 1. (a) multiple erythematous dome-shaped papules on the right auricle, (b) after total excision. 
Figure 2. (a) intersecting fascicles of spindled cells with intervening slit- and sieve-like vascular spaces surrounded by patchy lymphoplasmacytic infiltrate $(\mathrm{H} \& \mathrm{E} \times 10)$, (b) high power of intersecting fascicles with blood-filled, sieve-like vascular channels $(\mathrm{H} \& \mathrm{E} \times 20)$, positive immunoreaction for CD31 (c), CD34 (d) and HHV8 (e) which shows nuclear immunoreaction.

Table 1. Clinical features of external ear Kaposi sarcoma cases in this study and previous studies. 


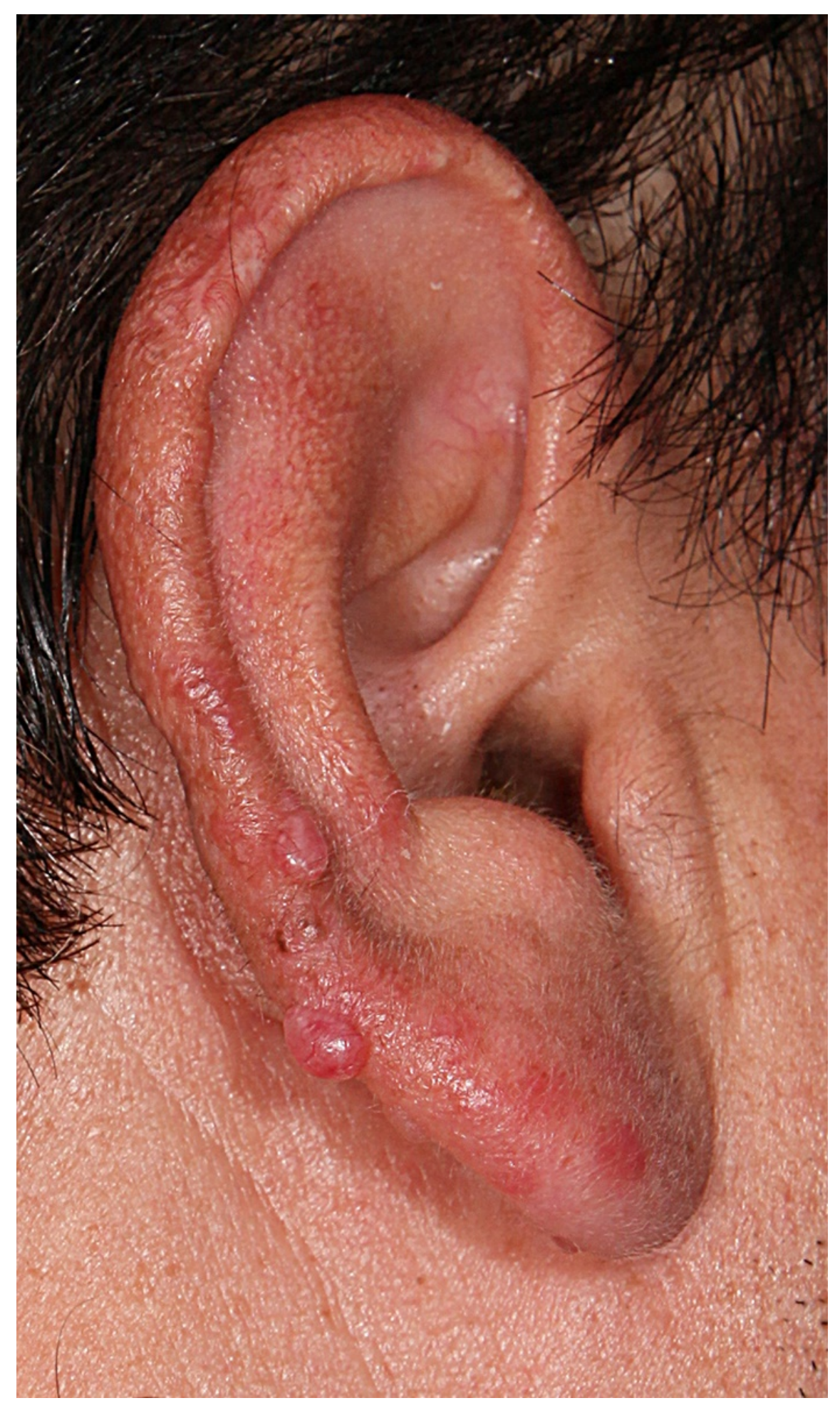




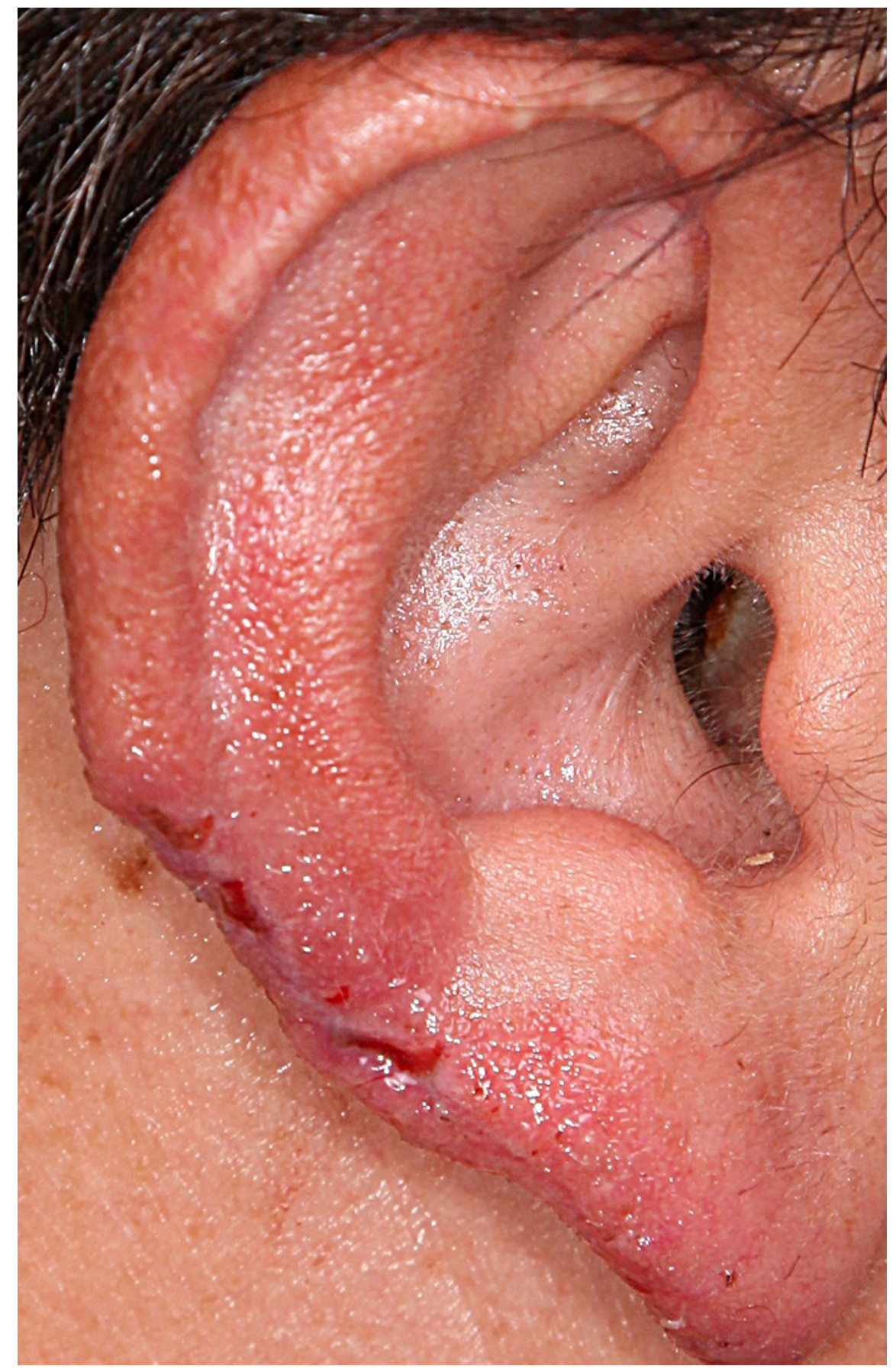



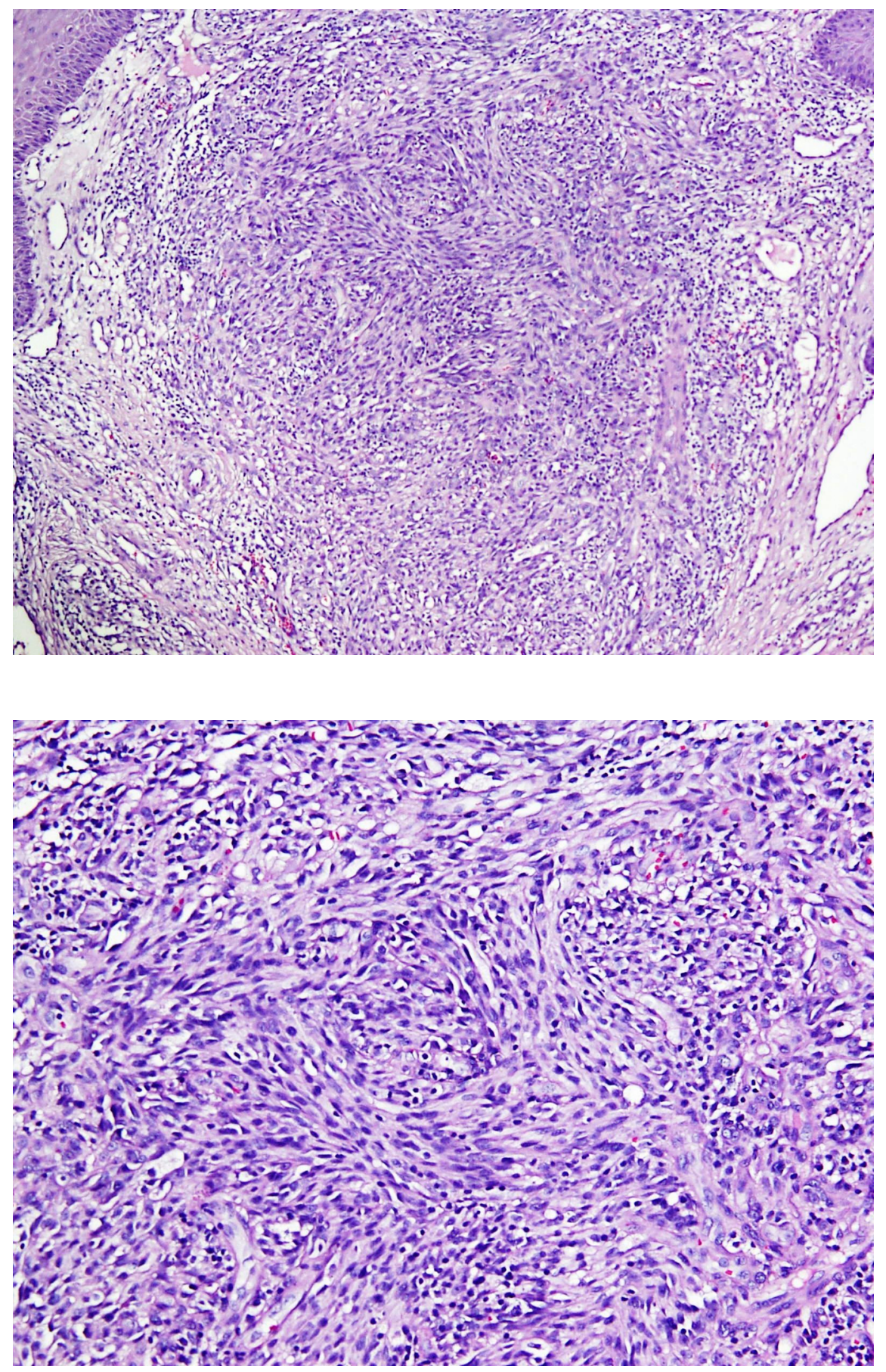

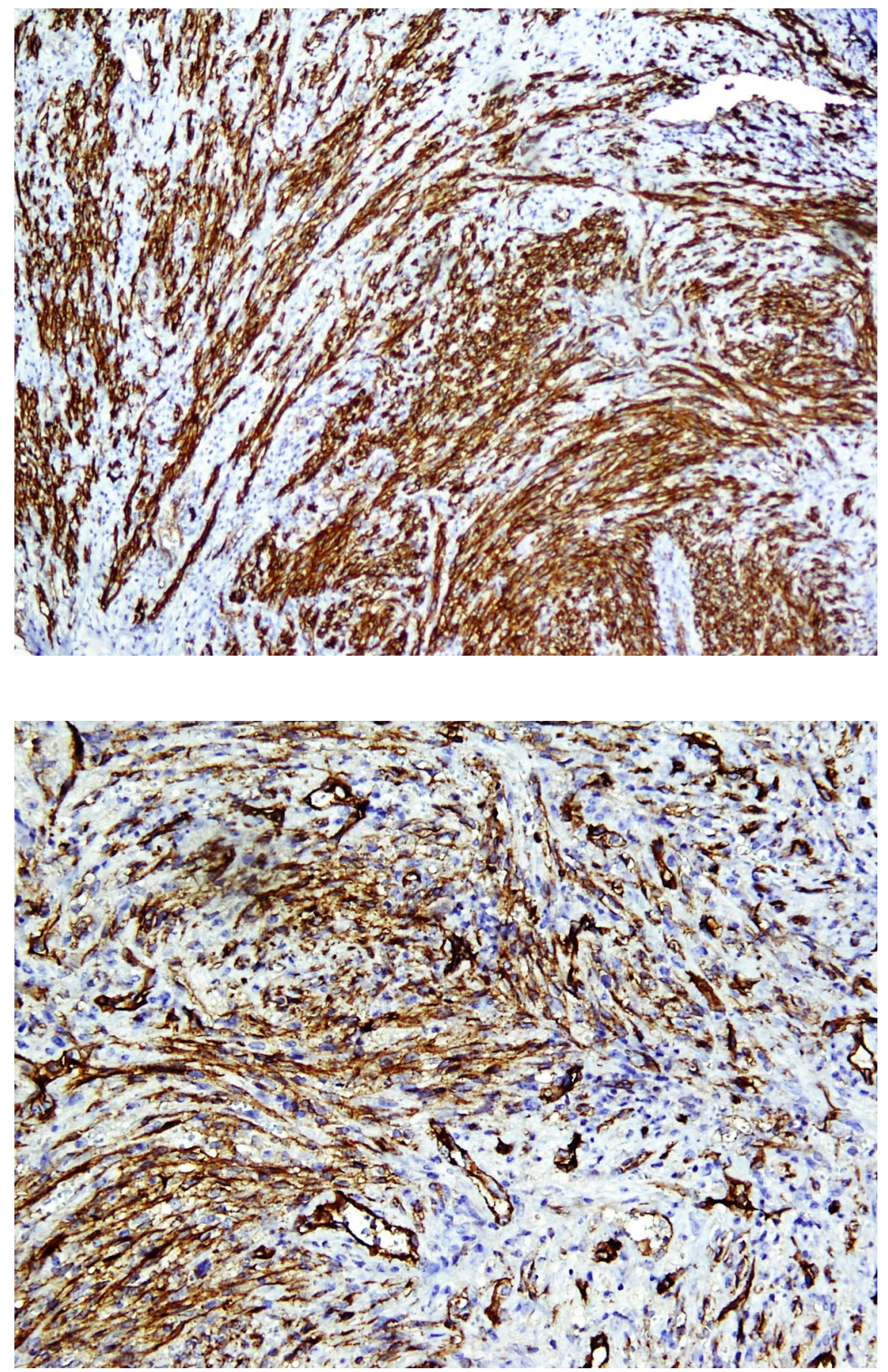


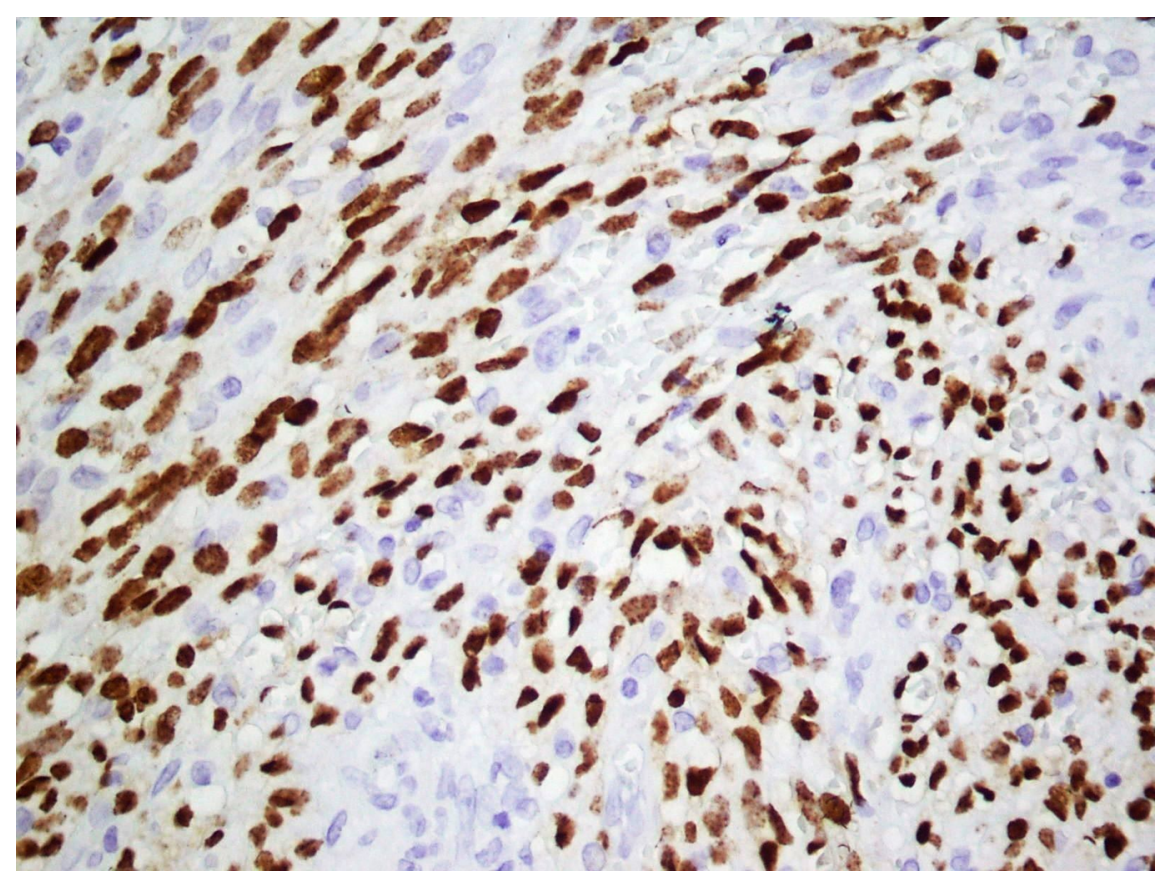

